\title{
Cirrhosis with Portal Hypertension in Pregnancy
}

\author{
${ }^{1}$ Mrityunjay C Metgud, ${ }^{2}$ Swati S Goudar, ${ }^{3}$ Kamal P Patil
}

\begin{abstract}
Background: Conception is a rare event in women with cirrhosis with portal hypertension due to altered metabolism of sex steroids and malnutrition, provided the liver disease is well compensated. Maternal prognosis is better with extrahepatic portal venous obstruction (EHPVO) and noncirrhotic portal fibrosis (NCPF) and poor with cirrhosis of the liver. Maternal mortality ranges between 2 and $18 \%$, being maximum with cirrhosis, whereas women with NCPF fare better with mortality rates between 2 and $6 \%$. Perinatal mortality ranges between 11 and $18 \%$, owing to preterm delivery or intrauterine growth restriction (IUGR). Nephrotic syndrome occurs in 0.012 to $0.025 \%$ of all pregnancies that add further insult to the uteroplacental insufficiency.
\end{abstract}

Aim: In the light of advancement of modern obstetrics, the rising incidence of pregnancy with cirrhosis and portal hypertension is of common occurrence with today's obstetricians who should be well equipped to deal with the various adverse maternal and fetal outcomes associated with this condition in coordination with other specialty branches for holistic management of the case.

Case report: A 35-year-old G3P1L1A1 known case of cirrhotic portal hypertension postsplenectomy 6 years earlier due to massive splenomegaly, severe anemia (pancytopenia) with immunoglobulin M (IgM) nephropathy, with previous baby having Down's syndrome presented at 20 weeks of gestation for further evaluation. Level II scan done was normal. Karyotyping of the fetus showed normal chromosomes. Upper gastroesophageal endoscopy done 2 years prior showed grade I esophageal varices. Ultrasonography abdomen showed heterogeneous echo texture of liver with cavernomatous transformation of the portal vein with extensive collaterals. The prognosis of this pregnancy was explained and continued with close monitoring with development of superimposed preeclampsia at 24 weeks of gestation. Urine routine and microscopy which showed albumin $3+, 80$ to 100 pus cells, and 50 to 60 red blood cells with casts, with serum albumin in liver function test (LFT) being 1.5 at 34 weeks of gestation. She was taken up for elective lower segment cesarean section (LSCS) at 35 weeks of gestation in view of superimposed preeclampsia with IUGR and a live male baby of $1.97 \mathrm{~kg}$ was delivered.

Conclusion: Patient had good pregnancy outcome despite having cirrhosis with portal hypertension and good renal function was achieved without steroids.

Clinical significance: A multidisciplinary team approach in tertiary care center with availability of intensive care units is

\footnotetext{
${ }^{1,3}$ Professor, ${ }^{2}$ Postgraduate Student

${ }^{1-3}$ Department of Obstetrics and Gynecology, Jawaharlal Nehru Medical College, KLE University, Belagavi, Karnataka, India

Corresponding Author: Swati S Goudar, Postgraduate Student, Department of Obstetrics and Gynecology, Jawaharlal Nehru Medical College, KLE University, Belagavi, Karnataka India, e-mail: swati.goudar90@gmail.com
}

likely to yield best pregnancy outcome in pregnant women with cirrhosis and portal hypertension despite various associated complications.

Keywords: Cirrhosis, Nephrotic syndrome, Pregnancy with portal hypertension.

How to cite this article: Metgud MC, Goudar SS, Patil KP. Cirrhosis with Portal Hypertension in Pregnancy. J South Asian Feder Obst Gynae 2018;10(2):134-137.

Source of support: Nil

Conflict of interest: None

Date of received: 15 March 2018

Date of acceptance: 20 April 2018

Date of publication: July 2018

\section{BACKGROUND}

The incidence of cirrhosis in pregnancy has been reported as 1 in 5,950 pregnancies. ${ }^{1}$ Pregnancy is rare in patients with cirrhosis as advanced liver disease does not typically occur until most patients have completed their families. Cirrhosis results in metabolic and hormonal derangements that lead to anovulation and amenorrhea. ${ }^{2}$ Malnutrition and altered hepatic metabolism of sex steroids may play a role but corroborative evidence is lacking. Fertility is normal or near normal in well-compensated cirrhosis, and infertility usually reflects the degree of hepatic dysfunction. ${ }^{3}$

The hemodynamic changes, in adaptation to the pregnancy and fetal needs, worsen the portal hypertension resulting in potentially life-threatening variceal bleed and others like severe anemia, hepatic decompensation, leading to progressive liver and renal failure, usually after variceal bleeding, hepatic encephalopathy, splenic artery aneurysm rupture, ascites, spontaneous bacterial peritonitis, and postpartum hemorrhage. ${ }^{4}$

Esophageal varices are seen in $>40 \%$ of the patients with cirrhosis at the time of diagnosis. Variceal bleed is noted in 18 to $32 \%$ of pregnant patients with cirrhosis and in $50 \%$ with associated portal hypertension. Highest risk of active variceal bleeding is commonly reported in the second and third trimesters of pregnancy and in second stage of labor.

Pregnant patients with cirrhosis may develop liver dysfunction in the form of jaundice, ascites, and/or hepatic encephalopathy in $24 \%{ }^{5}$

Maternal prognosis is better with EHPVO and NCPF and poor with cirrhosis of the liver. Maternal mortality 
ranges between 2 and $18 \%$, being maximum with cirrhosis; whereas women with noncirrhotic portal hypertension fare better with mortality rates between 2 and 6\%. ${ }^{6,7}$ The causes of death are generally hematemesis, hepatic coma, or postpartum hemorrhage. ${ }^{2}$

Perinatal mortality ranges between 11 and 18\%, owing to preterm delivery or IUGR. ${ }^{8,9}$ Of the women with cirrhosis, 20 to $30 \%$ will have hematemesis during pregnancy with the mortality ranging between 50 and $60 \% .{ }^{10}$ Nephrotic syndrome occurs in 0.012 to $0.025 \%$ of all pregnancies that adds further insult to the uteroplacental insufficiency with low colloid osmotic pressure and reduction in effective blood volume causing fetal growth restriction, prematurity, stillbirth, anasarca, polyhydramnios and acute kidney injury, chronic renal failure, gestational hypertension, and preeclampsia/ severe preeclampsia in the mother. ${ }^{11}$

\section{CASE REPORT}

A 35-year-old G3P1L1A1 known case of cirrhotic portal hypertension postsplenectomy 6 years earlier due to massive splenomegaly and severe anemia (pancytopenia) with IgM nephropathy with meshplasty done for incisional hernia 4 years earlier with previous baby having Down's syndrome presented at 20 weeks of gestation for further evaluation on August 04, 2015. Patient was a known case of cirrhotic portal hypertension 15 years prior to the present conception on Tab. Propranolol $5 \mathrm{mg}$ OD for control of portal hypertension in consultation with a gastroenterologist. She was investigated for various causes of cirrhosis leading finally to the diagnosis of autoimmune cirrhotic portal hypertension. Patient developed IgMnephropathy 2 years before this pregnancy, where close monitoring of renal profile with a nephrologist was normal.

Level II scan done was normal. Karyotyping of the fetus done in view of previous baby having Down's syndrome with no familial history showed normal chromosomes (Fig. 1). Upper gastroesophageal endoscopy done 2 years prior to present pregnancy showed grade I esophageal varices. Ultrasonography abdomen showed heterogeneous echo texture of the liver with cavernomatous transformation of the portal vein with extensive collaterals suggestive of cirrhosis of liver with portal hypertension. She developed superimposed preeclampsia at 24 weeks of gestation and was started on Tab. Methyldopa $500 \mathrm{mg}$ TID with good control of blood pressure. The routine antenatal investigations with liver and renal profile were within normal limits. The prognosis of this pregnancy was explained and continued with close monitoring.

Patient presented at 34 weeks of gestation with complaints of lower pain abdomen and burning micturition. On examination, vitals were stable, per abdomen uterus

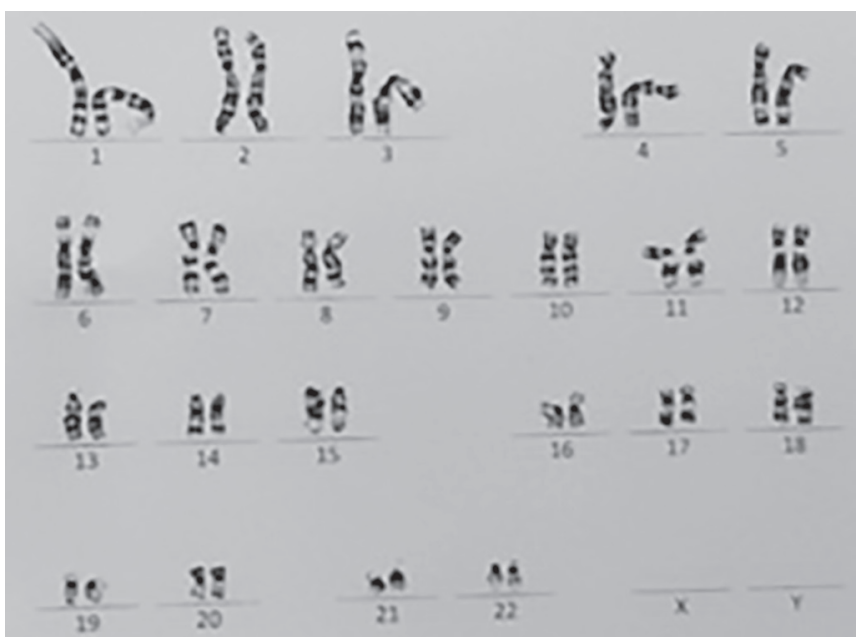

Fig. 1: Karyotyping report of the present fetus done at 20 weeks period of gestation in view of previous child with Down's syndrome showing normal karyotype of present pregnancy

was relaxed, fetal heart sounds was good with no cervical changes on per vaginal examination. Urine routine and microscopy which showed albumin $3+, 40$ to 60 pus cells and 10 to 20 red blood cells with granular casts and total proteins in LFT being 4.5, serum albumin of 1.5, and $\mathrm{A} / \mathrm{G}$ ratio reversal. Patient's complete hemogram, renal profile, and coagulation profile were normal. Obstetric scan showed IUGR with normal umbilical artery doppler profile.

The nonstress test was reactive and prophylactic antibiotics was started for urinary tract infection. With a gastroenterologist's opinion, two pints of albumin was transfused. Two doses $12 \mathrm{mg}$ betamethasone were administered 24 hours apart. In consultation with a nephrologist, gastroenterologist, maternal fetal medicine specialist, and a neonatologist, high-risk consent was obtained and the patient was taken up for elective LSCS at 35 weeks of gestation in view of cirrhosis with portal hypertension and superimposed preeclampsia and IUGR. A live male baby of $1.97 \mathrm{~kg}$ was delivered.

Liver examined intraoperatively appeared normal. Postpartum period was uneventful.

\section{DISCUSSION}

\section{Antenatal}

In the scenario of well-compensated liver disease, pregnancy is not contraindicated. There are various scoring systems in clinical practice to assess the severity of liver disease and these are basically used as a guide for allocation of organs in liver transplantation scores, the most widely used is the model for end-stage liver disease (MELD). Patients with a MELD score of 6 or less can be reassured of minimal significant complications and those with MELD score of 10 or above should be advised against pregnancy. ${ }^{12}$ 
Liver and renal function tests and hematological assessment should be done weekly till the completion of second trimester and then twice weekly. Close maternal and fetal monitoring by the joint team is recommended twice weekly from the third trimester and weekly until then. Effect of the drugs need to be carefully monitored both in the mother and the fetus.

Anemia should be treated emphatically as it leads to cardiac compromise in addition to being a risk factor for preterm labor, low birth weight. Since variceal bleed is the single most important complication linked with poor pregnancy outcome, the basic aim is to prevent it. Endoscopy being safe in pregnancy is the gold standard to assess the risk of bleeding in patients with esophageal varices. ${ }^{13}$

Nonselective beta-blockers are used to reduce portal pressure and also reduce the risk of first bleed by half, but the principal risk of using them in pregnancy is fetal growth restriction and fetal bradycardia. Current literature (Baveno V consensus workshop) recommends esophageal variceal ligation for acute esophageal variceal bleed, although endoscopic sclerotherapy may be used if banding is technically difficult. ${ }^{14}$ In case of failure to control bleeding endoscopically, emergency transjugular intrahepatic portosystemic shunt procedure may be needed. ${ }^{15}$

Aspirin or nonsteroidal anti-inflammatory drugs should be avoided which may precipitate hepatic decompensation and acute renal failure due to concurrent existence of cirrhosis with portal hypertension and nephrotic syndrome.

Pregnant women with cirrhosis are more prone to develop ascites with higher incidence of spontaneous bacterial peritonitis. Mortality rates are not high if treated early and adequately with third-generation cephalosporins. ${ }^{1}$

Pregnancy can be allowed to go to term if the disease is well compensated. In cases of progressive liver failure or any concurrent obstetric indication, early termination of pregnancy may be warranted. For planned termination before 34 weeks, antenatal corticosteroids can be administered for fetal lung maturity.

Cesarean section is usually reserved for the obstetrical indications. The Asian Pacific Association for the Study of the Liver (APASL) has developed consensus statement on various aspects of EHPVO, including pregnancy, and recommended that these women can be allowed for vaginal delivery. ${ }^{16} \mathrm{~A}$ short second stage of labour has been advocated along with consideration of forceps or vacuum, if needed. Although, the recognition that prolonged vaginal delivery with repeated valsalva manoeuvres would cause increased risk of variceal bleeding has recently led to an interest in considering caesarean section as an alternative. There is no data comparing the outcomes of pregnant women with cirrhosis with portal hypertension with caesarean versus vaginal delivery and the optimal obstetric outcome has to be individualized to each patient based on co-existing maternal and fetal co-morbidities such as our case. ${ }^{17}$

\section{Peripartum}

Adequate amount of blood and plasma should be arranged and measures for balloon tamponade for the variceal hemorrhage must be handy. Second stage of labor must be shortened to avoid overstraining efforts of the mother. Epidural analgesia is the preferred choice as it can also work in case cesarean is required. ${ }^{1}$ The third stage should be managed actively and methergine should be avoided among the oxytocics.

The rates of spontaneous abortion, premature birth, still births, and perinatal death are increased in women with portal hypertension. There is 10 to $66 \%$ perinatal mortality in patients of liver cirrhosis and spontaneous abortion rate of about $20 \%$ in the first trimester. ${ }^{18,19}$

\section{Postpartum}

Antibiotic use needs to be individualized with spontaneous bacterial peritonitis in mind for patients with cirrhosis of liver. Breast feeding is usually not contraindicated in these women unless she is on Food and Drug Administration category $\mathrm{D}$ or $\mathrm{X}$ drugs.

Reliable contraception must be advised in the form of barrier methods, intrauterine devices, or permanent sterilization. Permanent sterilization may not be possible due to coagulopathy, and hormonal contraception is avoided as it may cause cholestasis. ${ }^{1}$

\section{CONCLUSION}

Patient had good pregnancy outcome and good renal function was achieved without steroids with the coordinated and combined team management by related specialty branches with all measures kept ready to deal with any obstetric emergency and the availability of intensive care unit if need be.

Prognosis of patients with cirrhotic portal hypertension is poorer than noncirrhotic portal hypertension which is further worsened by addition of other multisystem complications like nephrotic syndrome and superimposed preeclampsia.

\section{CLINICAL SIGNIFICANCE}

Bearing in mind the various adverse maternal and fetal outcomes, some of whose expert management comes into the premise of other superspecialty branches, a multidisciplinary team approach in tertiary care center with 
availability of intensive care units is likely to yield best pregnancy outcome in pregnant women with cirrhosis and portal hypertension despite various associated complications.

\section{ACKNOWLEDGMENTS}

Authors would like to thank the following:

- Dr Hema Dhumale, Professor of Obstetrics and Gynecology, Department of Obstetrics and Gynecology, Jawaharlal Nehru Medical College, Belagavi, Karnataka, India, for her contribution with expertise in advanced obstetric ultrasonography.

- Dr Rajesh Mane, Professor of Anesthesia, Department of Anesthesia, Jawaharlal Nehru Medical College, Belagavi, Karnataka, India, for careful consideration of anesthesia and intensive intraoperative monitoring.

- Dr Santosh Hazare, Professor and Head of Gastroenterology, Department of Gastroenterology, Jawaharlal Nehru Medical College, Belagavi, Karnataka, India, for his valuable input toward the management of cirrhosis and portal hypertension.

- Dr MS Korishetti, Professor and Head of Nephrology, Department of Nephrology, Jawaharlal Nehru Medical College, Belagavi, Karnataka, India, for his decisive treatment of nephrotic syndrome in pregnancy.

- Dr Sangappa Dhaded, Professor and Head of Neonatology, Department of Pediatrics and Neonatology, Jawaharlal Nehru Medical College, Belagavi, Karnataka, India, for his expert intensive neonatal care and monitoring leading to a fruitful outcome.

- Dr Jayprakash Appajigol, Professor of Medicine, Department of General Medicine, Jawaharlal Nehru Medical College, Belagavi, Karnataka, India, for his supportive care.

\section{REFERENCES}

1. Peitsidou A, Peitsidis P, Michopoulos S, Matsouka C, Kioses E. Exacerbation of liver cirrhosis in pregnancy: a complex emerging clinical situation. Arch Gynecol Obstet 2009 Jun;279(6): 911-913.

2. Sowjanya R, Sudha R. Cirrhosis with portal hypertension in pregnancy. IOSR J Dent Med Sci 2015 Apr;14(4):10-11.
3. Fagan, EA. Disorders of the liver, biliary system and pancreas. In: de Sweit M, editor. Medical disorders in obstetric practice. 4th ed. Oxford: Blackwell Science; 2002. p. 322.

4. Aggarwal N, Negi N, Aggarwal A, Bodh V, Dhiman RK. Pregnancy with portal hypertension. J Clin Exp Hepatol 2014 Jun;4(2):163-171.

5. Benjaminov FS, Heathcote J. Liver disease in pregnancy. Am J Gastroenterol 2004 Dec;99(12):2479-2488.

6. Cheng YS. Pregnancy in liver cirrhosis and/or portal hypertension. Am J Obstet Gynecol 1977 Aug;128(7):812-822.

7. Russell MA, Craigo SD. Cirrhosis and portal hypertension in pregnancy. Semin Perinatol 1998 Apr;22(2):156-165.

8. Shaheen AA, Myers RP. The outcomes of pregnancy in patients with cirrhosis: population based study. Liver Int 2010 Feb;30(2):275-283.

9. Aggarwal N, Sawhney H, Vasishta K, Dhiman RK, Chawla Y. Noncirrhotic portal hypertension in pregnancy. Int J Gynaecol Obstet 2001 Jan;72(1):1-7.

10. Lee WM. Pregnancy in patients with chronic liver disease. Gastroenterol Clin North Am 1992 Dec;21(4):889-903.

11. Pandya BK, Gibson SP, Robertson IG. Nephrotic syndrome in early pregnancy-is renal biopsy always necessary? Nephrol Dial Transplant 2002 Apr;17(4):672-674.

12. Westbrook RH, Yeoman AD, O'Grady JG, Harrison PM, Delvin J, Heneghan MA. Model for end-stage liver disease score predicts outcome in cirrhotic patients during pregnancy. Clin Gastroenterol Hepatol 2011 Aug;9(8):694-699.

13. O'Mahony S. Endoscopy in pregnancy. Best Pract Res Clin Gastroenterol 2007;21(5):893-899.

14. de Franchis R; Baveno V faculty. Revising consensus in portal hypertension: report of Baveno $\mathrm{V}$ consensus workshop on methodology of diagnosis and therapy in portal hypertension. J Hepatol 2010 Oct;53(4):762-768.

15. Lodato F, Cappelli A, Montagnani M, Colecchia A, Festi D, Azzaroli F, Compagnone G, Cecinato P, Golfieri R, Mazzella G. Transjugular intrahepatic portosystemic shunt: a case report of rescue management of unrestrainable variceal bleeding in a pregnant woman. Dig Liver Dis 2008 May;40(5):387-390.

16. Sarin SK, Sollano JD, Chawla YK, Amarapurkar D, Hamid S, Hashizume M, Jafri W, Kumar A, Kudo M, Lesmana LA, et al. Consensus on extra-hepatic portal vein obstruction. Liver Int 2006 Jun;26(5):512-519.

17. Tran T, Ahn Joseph, Reau Nancy. ACG Clinical Guideline: Liver disease and Pregnancy. doi:10.1038/ajg.2015.430.

18. Britton RC. Pregnancy and esophageal varices. Am J Surg 1982 Apr;143(4):421-425.

19. Varma RR, Michelson NH, Borkowf HI, Lewis JD. Pregnancy in cirrhotic and non cirrhotic portal hypertension. Obstet Gynecol 1977 Aug;50(2):217-222. 